\title{
Fluid Structure Interaction of Hydrofoils
}

\author{
Dr. Jason Knight \\ University of Portsmouth \\ Portsmouth, U.K. \\ jason.knight@port.ac.uk
}

\author{
Tony Ledger, Thales Group, Reading \\ Matthew McConnell, University of Portsmouth \\ Dr. Rodrigo Azcueta, Cape Horn Engineering, Portsmouth
}

\section{Synopsis}

Hydrofoils are used in the marine industry to produce enough lift to raise the boat and crew out of the water, therefore reducing resistance on the hull and enabling increased speeds. The interaction between the hydrofoil and water puts severe stress and strain on the hydrofoil. Fluid-structure interaction (FSI) is a multi-physics coupling of both fluid dynamics and structural mechanics into one simulation. When a fluid flow interacts with a structure, stresses and strains are applied within the structure which can lead to a deformation, which can change the flow field, giving a revised pressure loading. This change in pressure loading can lead to either an increase or decrease in lift, which is dependant on the location of the elastic axis of the hydrofoil. If the pressure loading is increased and left unchecked, the deformation could lead to failure of the structure.

A symmetrical hydrofoil is studied and good agreement to within $1 \%$ variation in pressure is found between the simulated fluid and experimental results found in literature. Good agreement is essential for FSI as any differences can be amplified in subsequent iterations of the FSI. The FSI effects of lift are reported with varying material properties for the NACA0012 hydrofoil. The lift was found to be highly dependent on structural rigidity. The FSI effects are reported for a particular case with a tip deflection of $45 \mathrm{~cm}$ which is $23 \%$ of span. This results in an increase of lift by a factor of $19 \%$, although much larger deformations are possible.

In addition, the effects of an FSI on the more complex geometry of the daggerboard on the AC45F foiling boat used in America's Cup are presented. Here, due to FSI effects, the tip deflection of $32 \mathrm{~cm}$ changes the coefficient of lift by a factor of $10 \%$. All FSI simulations are found to be stable and give an indication of material strengths needed. However, in all analyses we simplify the structural simulation by treating the structure as a solid volume with isotropic material properties. Future work including the use of anisotropic material properties are highlighted.

Keywords - Fluid-Structure Interaction, drag reduction, flow resistance, hydrofoils.

\section{Introduction}

Fluid-structure interaction (FSI) is a multiphysics coupling of both fluid dynamics and structural mechanics into one simulation. When a fluid flow interacts with a structure, stresses and strains are applied to the structure, which can lead to a deformation. The resulting deformation can give a revised fluid flow, which in turn can lead to a revised deformation (Knight, 2010).

Hydroelasticity is a relatively new area of research. However, it is similar to aeroelasticity, which is a term associated with a group of problems predominantly within aircraft design. Aircraft wings have some flexibility within them, which leads to structural deformations where the aerodynamic forces upon the structure can increase. This greater increase of aerodynamic forces will induce even more structural deformation, which could lead to destruction of the structure, unless a stable equilibrium has been reached (Bisplinghoff, Ashley, \& Halfman, 1955). This is known as divergence, which is an aeroelastic as well as a hydro-elastic effect. Other aeroelastic effects include control reversal and flutter (Arioli \& Gazzola, 2017; Panda \& SRP 2009). Divergence and control reversal are known as static 
aero-elastic effects, whereas flutter is a dynamic aero-elastic effect.

Solving an FSI problem such as divergence involves combining the two independent computational models of the FEA and CFD together, which due to their independency from one another, can cause complications. To enable the two models to efficiently transfer data, the type of application needs to be considered. The approach to model an FSI can be categorised in three main strategies, structural models can either be fully, closely or loosely coupled with the fluid models (Tu, Yeoh, \& Liu, 2013). Both the fluid and structural equations are combined in a fully coupled technique and solved simultaneously in respect to time. Limitations of this method exist due to the different reference systems used between both FEA and CFD codes and their interaction between each other (Crouch, 2016). An Eulerian approach is usually used in the fluid equations which is combined with the Lagrangian approach in the structural equations (Kamakoti \& Shyy, 2004), whereby an interface is generated to enable data transfer (Sigrist, 2015). Closely coupling the two codes requires a similar boundary interface, but with this method the interface is mapped. The mesh can morph according to the pressure from the fluid, which is used by the structural model and then changed into a displacement. The loosely coupling method has no interface between the two FEA and CFD codes, with the equations solved independent of each other with two independently discretised grids. This is the simplest method and the approach we use in this work.

Research in hydro-elastic response and stability of hydrofoils in viscous flow has been conducted by Ducoin and Young (2013). They validated their work against experimental measurements for a NACA0012 hydrofoil and then progressed onto the NACA66 hydrofoil. They compared numerical models to identify the instability of the hydrofoils and the static stalls associated with this instability using a loosely coupled FSI method. The results for the 3 different Reynolds numbers investigated seem to give good correlation between the simulated results and experimental results, except for when the Reynolds number is 660,000. At high angles of attack the stalling of the hydrofoil leads to discrepancies between the results, due to the separation of the flow.
We use a similar approach to Ducoin and Young (2013) and validate our work using a 2D simulation on a NACA0012 aerofoil by comparing results with literature. Then progressing with comparisons using quasi-2D mesh and also 3D mesh with tip exposed to flow and unconstrained. We investigate the effect of material properties in the fluid structure interaction. Thereafter the AC45F Daggerboard is investigated using a similar setup to that validated. Finally, methods of how to reduce the FSI effects are discussed and proposals identified for future work.

\section{Methodology}

\subsection{Symmetrical 2D wing}

The NACA0012 aerofoil is used to provide confidence and accuracy in the CFD simulation results before we progress onto the more complex geometry of the daggerboard. This NACA0012 aerofoil was chosen because of the large amount of experimental data available (Ramsay, 2006). For comparison to wind tunnel results conducted by NACA, ISA Standard conditions are used with the Reynolds Number of 6 million. The aerofoil itself will be treated as a non-slip wall boundary condition. The domain has velocity inlet and pressure outlet. All remaining boundaries are defined as symmetry planes. The Spalart-Allmaras Turbulence model is used initially for the validation case both in 2D and quasi-2D simulations.

The chord length of the aerofoil was set as $1 \mathrm{~m}$ and the far field domain was constructed using 5 chord lengths for the front, above, below and 10 for the rear. To capture the wake region with more fidelity, 2 geometric blocks are overlaid which encapsulates the aerofoil and its wake. Default controls for the mesh operation with the following values are used, $0.25 \mathrm{~m}$ base size, $2 \%$ for the target size and $0.5 \%$ of the base size for the minimum size. 15 prism layers were used with a thickness of $1 \%$ of base size and stretched using the ratio of 1:5. Two volumetric controls are created for the meshing process, having a custom size of $15 \%$ and $10 \%$ of the base size respectively. The resultant mesh can be seen in Figures 1a-d. The simulations typically ran for 2000 iterations and all converge below 1e-04 value. The convergence plot using an angle of $10^{\circ}$ is shown in Figure 2 and was typical for low angles of attack. Higher angles of attack required more iterations to converge. 

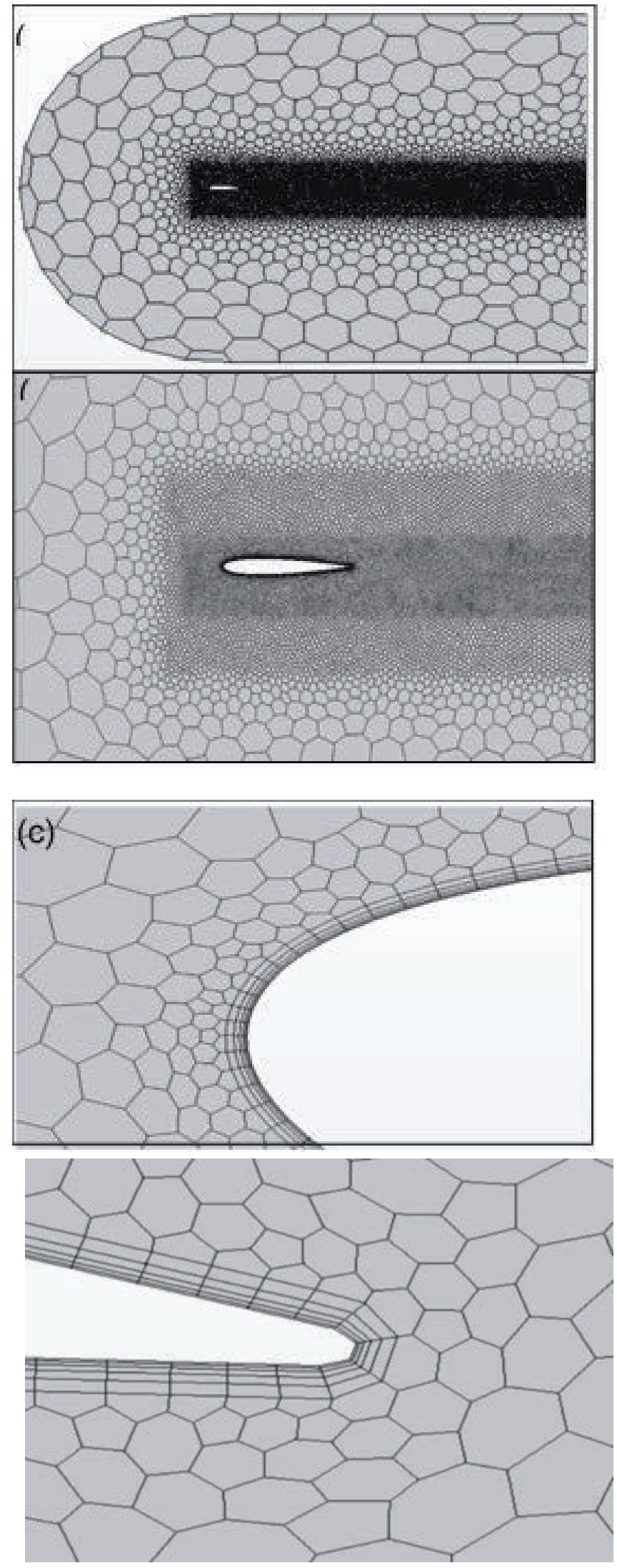

Figure 1: Mesh for 2D aerofoil, (a) overall domain (b) aerofoil (c) leading edge (d) trailing edge

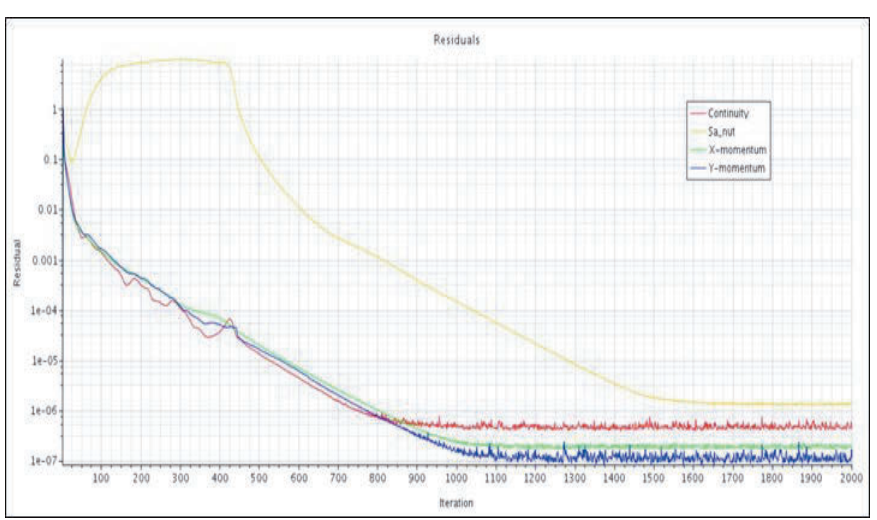

Figure 2: Typical residual plot showing convergence at 1500 iterations

Meshing is known to be a cause of many errors within CFD analysis (Versteeg \& Malalasekera, 2007; Zikanov, 2011). The solution itself can be driven by the mesh. Therefore, a mesh independency study is undertaken. This type of study shows that no matter how many extra cells you add into the simulation domain, the final converged result will be within an acceptable level of error. This process optimises the mesh reducing computational power and time to converge (Anderson, 1995). A mesh independency study was conducted for the 2D wing and is shown in Figure 3 , which shows the coefficient of lift against the number of cells within the mesh. With 70,000 cells the value of $\mathrm{Cl}$ plateaus, meaning that if a mesh consisted of approximately 25,000 cells - where the line reaches maximum value, the time to completion is reduced significantly but provides a suitable value of $\mathrm{Cl}$ within $2 \%$.

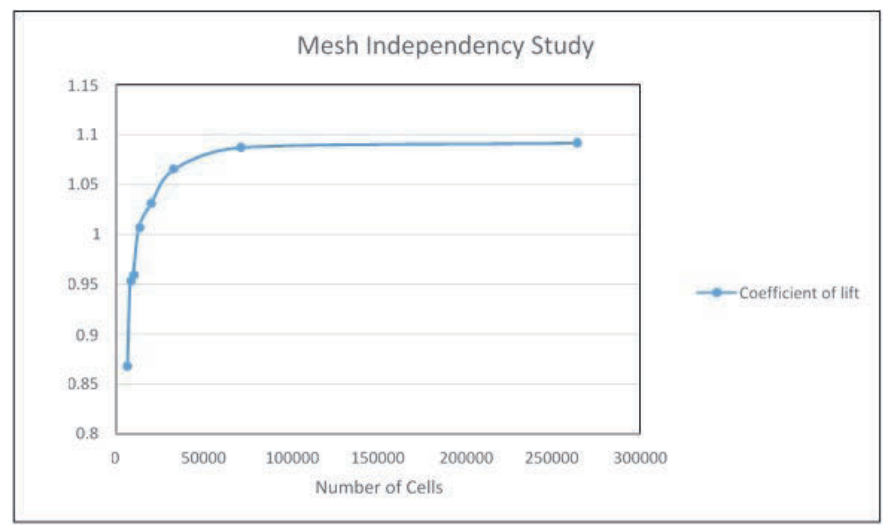

Figure 3: Mesh independency study with coefficient of lift against number of cells. 


\subsection{Quasi-2D aerofoil (3D)}

The quasi-2D simulation uses a similar resolution to that used in the 25,000 cells $2 \mathrm{D}$ simulation. The domain and wing are both extruded along the spanwise axis by $0.5 \mathrm{~m}$, which resulted in increasing the mesh size to 382,000 cells. The range of Wall Y+ values are all below 2.2 as shown in Figure 4.

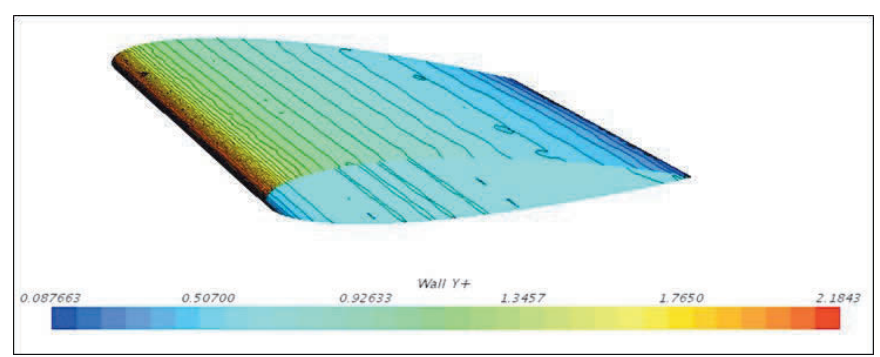

Figure 4: Wall Y+ values of aerofoil showing maximum value of 2.2 at leading edge

At the same angle of attack of 10 degrees, the 2D simulation using 25,000 cells and quasi-2D simulation with 382,000 cells have been plotted against the wind tunnel tests obtained from NACA and are seen in Figure 5.

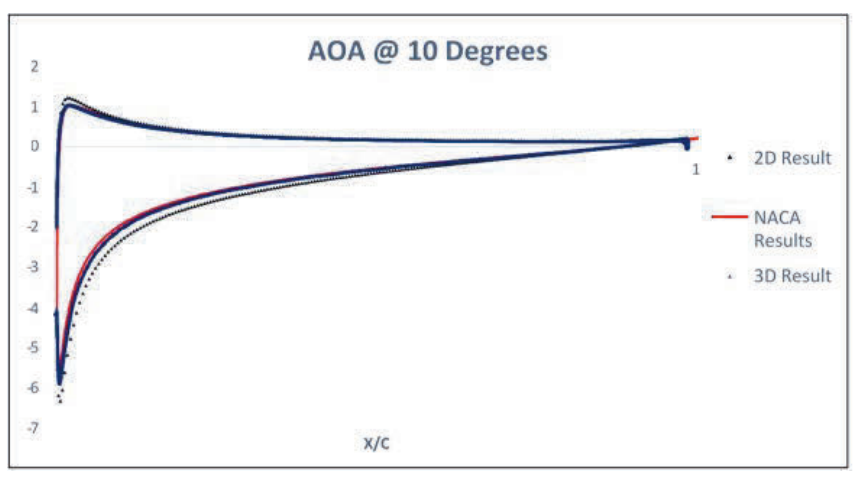

Figure 5: Pressure coefficient of 2D and 3D simulations against NACA experimental results at $10^{\circ}$ angle of attack

The 2D simulation overpredicts the minimum and maximum pressures when compared to the experiment. The pressures between the experimental result and quasi-2D simulation (3D) with 382,000 cells are indistinguishable, as can be seen in Figure 5. The pressure distributions show good agreement and the $\mathrm{y}^{+}$is within the recommended range. The $\mathrm{Cl}$ and $\mathrm{Cd}$ are calculated to be 1.08 and 0.0457 respectively. The lift correlates well with results widely found in literature around 1.07, whereas the drag is overpredicted when compared to values around 0.02 . Nevertheless, we conclude that the validation for the NACA0012 in air to be sufficient for our purpose due to the good agreement found in pressure between both simulation data and experimental results, noting that the lift is the dominant force in FSI. This validation has been made with air using the Spalart-Allmaras Turbulence model to facilitate close comparison with literature. Thence, ensuring our methodology correlates well and is reasonably accurate before proceeding. Hereafter, we change the fluid to water and use the more advanced SST K- $\omega$ turbulence model.

\subsection{Fluid-Structure Interaction (FSI) of 3D Wing with Tip Exposed}

The quasi-2D simulation previously used is modified by further extending the domain in the spanwise direction. The span of the wing is extended to $2 \mathrm{~m}$ and the wing tip is exposed to the flow field. This allowed the aerofoil to experience wing tip effects due to the vortices produced. We change to the SST K- $\omega$ turbulence model for an improved prediction of the wing tip vortices. The mesh size for this simulation is 421,898 cells arranged with a slightly reduced wake refinement condition around the aerofoil. The pressures and wall shear stresses are passed to the structural FEA code by using the two-way loosely coupled technique. These values are used to derive displacement, which is then transferred back to the CFD code. A mesh continuum for the hydrofoil was used to discretize it for the FEA solver. A mesh dependency study was also performed on the structural mesh. It became a linear tetrahedron which has 4 nodes and gave an accurate solution due to the stresses not changing rapidly and no locking of the elements. A higher resolution was used near the root of the wing. Only 20,500 cells were found to accurately describe the motion. Two motion methods are created, solid displacement and the morphing motion, which are assigned to the foil and fluid respectively. Allowing the two-way coupling needed for the FSI simulation. The creation of a vector warp of the aerofoil, hydrofoil and daggerboard allowed the displacement to be visualised, so a comparison to the original position can be easily made. 


\section{Results}

\subsection{Symmetrical wing}

Having validated our quasi-2D simulation with air, we now progress onto using water as the fluid and set the inlet speed to be 30 Knots. We initially use a strong and inflexible material to provide small deformations to test our approach with the NACA0012 hydrofoil. The hydrofoil is defined with material properties of Carbon Steel UNSG10100, which are $7832 \mathrm{~kg} / \mathrm{m}^{3}, 200 \mathrm{GPa}$ and 0.285 for the density, Young's Modulus and Poisson's Ratio respectively. The hydrofoil contains 20,500 structural elements. The overall displacement for the hydrofoil can be seen in Figure 6 , which has been amplified in the vertical plane.

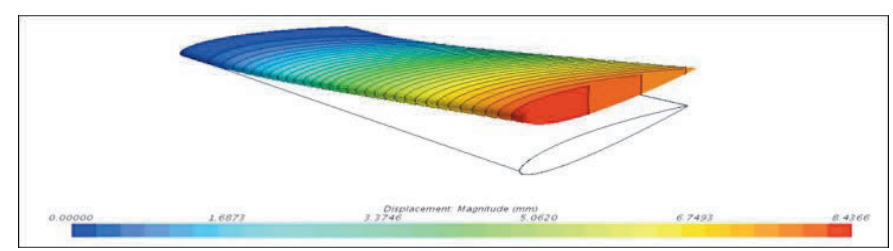

Figure 6: Displacement magnitude measured in $\mathrm{mm}$ of NACA0012

The tip of the leading edge has been displaced the most by the fluid, with a value of $8.4 \mathrm{~mm}$, which is less than $0.5 \%$ of the wingspan. The trailing edge is displaced slightly less at approximately $7 \mathrm{~mm}$. The wall $\mathrm{Y}+$ was in the range 0.34 to 3.5 and so within limitations of the model. This gave a value for the $\mathrm{Cd}$ of 0.048 for the rigid wing increasing to a new value of 0.0483 for the deformed wing. The $\mathrm{Cl}$ began with a value of 0.63 and increased to 0.635 . Both of these increases are less than $1 \%$ and so have a negligible overall effect.

Having achieved our FSI with an inflexible material, we now investigate the FSI with reduced material properties. The value of the Young's Modulus is reduced by a factor of 40 to $5 \mathrm{GPa}$. The hydrofoil can be seen to deflect much more as indicated in the legend of Figure 7.

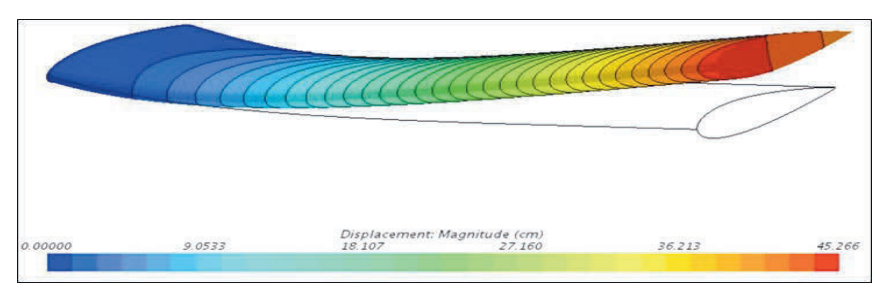

Figure 7: Displacement of NACA0012 with Young's Modulus of $5 \mathrm{GPa}$
The maximum displacement is $450 \mathrm{~mm}$ at the leading edge of the wing tip. The trailing edge has a slightly reduced deflection of approximately $440 \mathrm{~mm}$. This is similar behavior to that seen in the earlier computation with the less flexible wing as can be seen when comparing Figures 6 and 7 . Again, the $\mathrm{Cl}$ for the rigid wing starts at approx. 0.63 but increases to 0.76 due to the larger increase in angle of attack. The $\mathrm{Cd}$ starts at 0.048 increasing to 0.64 . The overall result to the $\mathrm{Cl}$ and $\mathrm{Cd}$ can be clearly seen to change due to this deflection. There is a $19 \%$ increase of $\mathrm{Cl}$, before and after deflection and a larger $33 \%$ increase in the $\mathrm{Cd}$ due to the fluidstructure interaction. These results represent the extremes shown in Figure 8. Further analysis on hydrofoil whilst at the same $10^{\circ}$ AOA using a variety of Young's Modulus shows in more detail the interaction of both fluid and the solid used. Figure 8 shows the Young's Modulus for the hydrofoil, with the value of both $\mathrm{Cl}$ and $\mathrm{Cd}$ reported before and after the deflection of the structure.

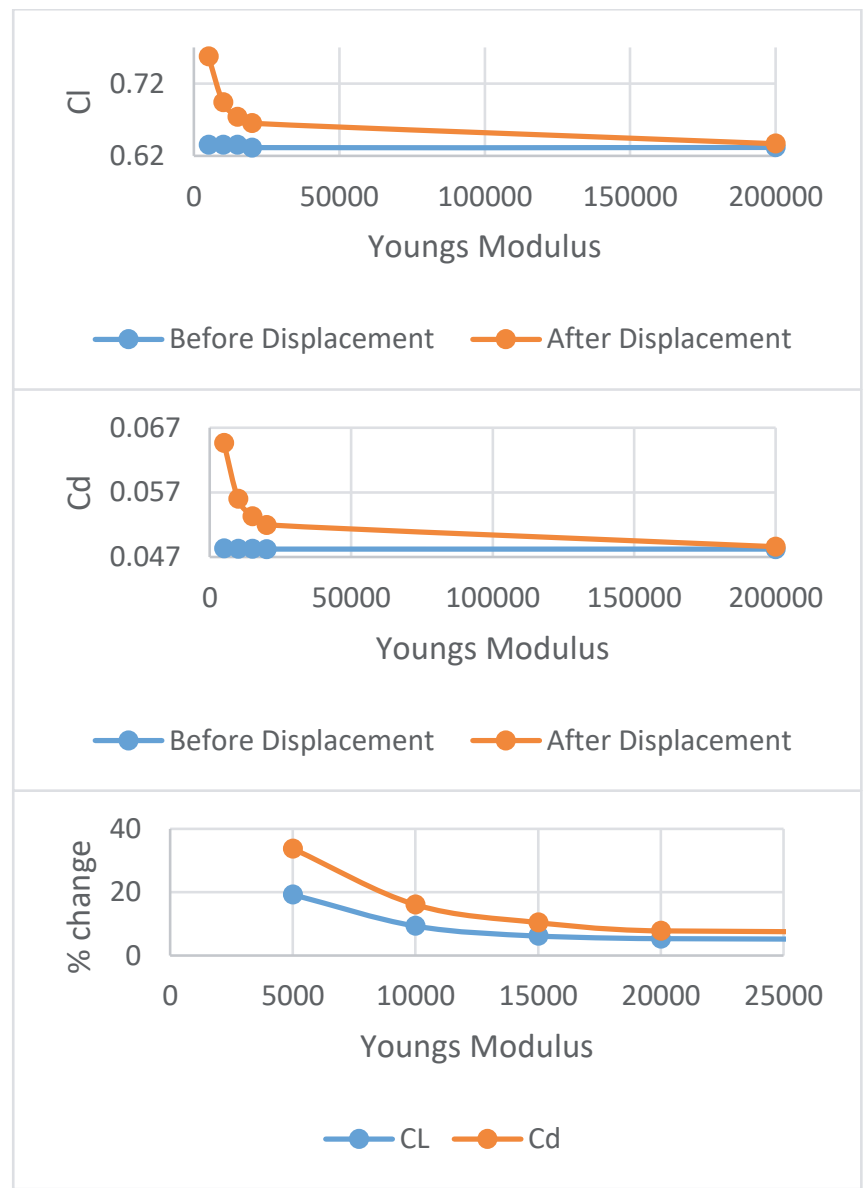

Figure 8: Coefficient of (a) lift and (b) drag of NACA0012 at $10^{\circ} \mathrm{AOA}$ and (c) \% changes in lift and drag due to FSI with varying Youngs Modulus 
As can be seen in Figure 8, reducing the Youngs Modulus increases the lift and drag due to the increased deformation of the hydrofoil. These increases appear linear, albeit with sparse data, until a point when they increase rapidly, possibly indicating the start of divergence. A further reduction in material properties or increase in speed is likely to result in failure of the hydrofoil.

\subsection{Daggerboard}

The AC45F class catamaran uses hydrofoils which raise the boat out of the water, reducing the overall drag and allowing for speeds of the boat up to 40 knots. This hydrofoil is made up of woven carbon fibre with $40 \%$ matrix, in various lay directions to increase the rigidity and help the foil maintain its shape and properties under these immense forces. It is therefore a complex piece of engineering. Carbon fibre is an anisotropic material. However, for the purposes of this study, the material will be treated as an isotropic solid body.

Importing the geometry into Star-CCM+, surfaces of the hydrofoil are designated by their dry and wet elements, as the hydrofoil itself is not fully submerged when in use. Figures $9 a$ and $b$ show how the hydrofoil is situated within the fluid domain for the simulations. The domain is $11 \times 11 \times 25 \mathrm{~m}$ in dimension with the hydrofoil sitting $6 \mathrm{~m}$ from the inlet. The meshed surface of the hydrofoil is shown more clearly in Figure 9c.
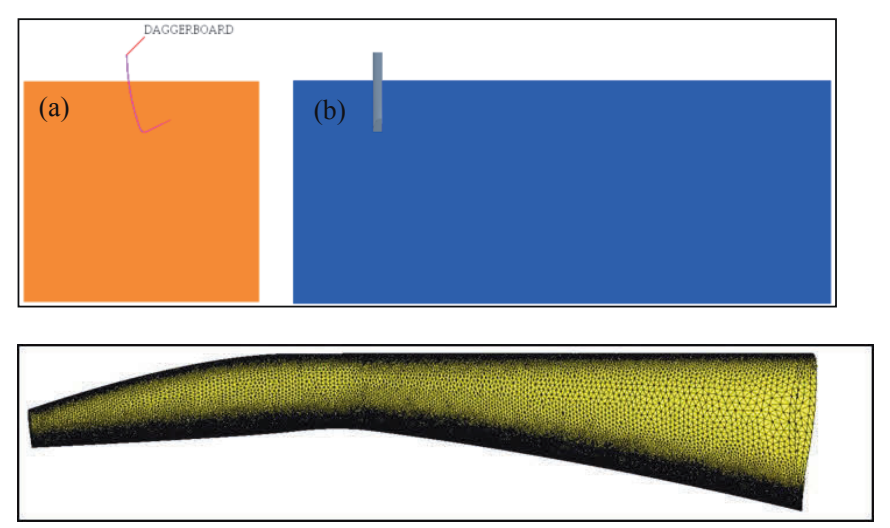

Figure 9: Domain surrounding daggerboard (a) view from inlet (b) side view (c) surface mesh.

Using a flow speed of 30 knots, the pressure in the plane cross-section of the daggerboard can be seen in Figure 10. Under this loading, the daggerboard is under a lot of stress and the overall displacement of the daggerboard is shown in Figure 11 whereby the maximum displacement can be seen to be $32 \mathrm{~cm}$ at the tip.

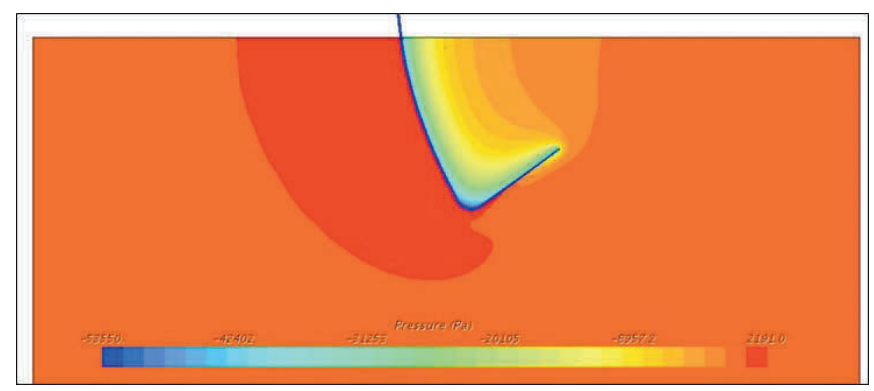

Figure 10: Pressure field surrounding daggerboard in $\mathrm{Pa}$.

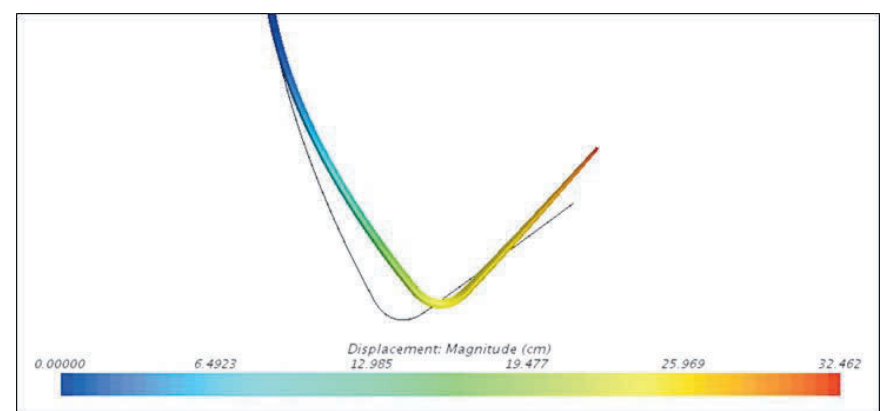

Figure 11: Displacement magnitude of daggerboard in $\mathrm{cm}$.

The maximum deflection of the daggerboard with the isotropic material properties assigned is $32 \mathrm{~cm}$ in magnitude and the calculated twist is leading edge upwards by approximately $1^{\circ}$. Initially for the daggerboard, the $\mathrm{Cl}$ and $\mathrm{Cd}$ are 0.265 and 0.0217 respectively. When applying the material properties of carbon fibre with $40 \%$ matrix, allows the pressure from the fluid to be passed onto this material type and then cause a deflection resulting in the revised fluid flow where the new $\mathrm{Cl}$ and $\mathrm{Cd}$ of $0.299,0.0218$ respectively are found. Thence the lift has increased by approximately $11 \%$ with a negligible increase in drag. Here, the increase in lift is mostly due to the hydrofoil deforming to have a larger horizontal or plan area. Whereas, in the validation case using the NACA0012, the increase in lift was due to twist alone. The displacement of the daggerboard can clearly be seen in Figure 11 and is significant, whereas the twist is relatively small. This leads to a small change in pressure field and so only 2 iterations are needed between fluid and structural compuations to reach the converged solution shown in Figure 11.

Experimental validation is very important to prove conclusively the accuracy on any FSI. Unfortunately no experimental results are available 
for the daggerboard. However, the methodology and mesh resolutions in both the validation case with NACA0012 and daggerboard were very similar. Thence, the authors believe the results to be realistic for the daggerboard although we acknowledge, it is not fully proven.

\section{Conclusion}

Good agreement has been found with our simulated results when compared with experimental results for NACA0012. This validation is a pre-cursor to an accurate FSI simulation. The FSI response was captured successfully with the symmetrical hydrofoil and the more complex shape of the daggerboard. The effect of material properties has been shown to be highly influential on the overall displacement and corresponding increase in lift. This can manifest itself by changes in twist as in the validation case or by changes in effective area as in the daggerboard case. Hence, two mechanisms are highlighted when investigating FSI and both are reliant on material properties characteristics.

Changing the Young's Modulus gives a good indicator on how a structure deforms and imparts a velocity on to the fluid providing a revised fluid flow. This allows a representation of the structure's behaviour when given certain material parameters. However, modelling in this linear fashion, whereby the material choice is limited to values of; Young's Modulus, density or the Poisson's ratio, limits the material type in question in which is trying to be investigated. Composites such as carbon fibre are of real interest due to how lightweight and stiff they can be manufactured and in specified directions. Further work to investigate how the daggerboard behaves with anisotropic material properties would be of specific interest. In addition, the position of the elastic axis can be varied and will have a significant effect on the FSI. Identifying the transient response of the daggerboard where time is a factor would also be further work of interest.

The use of FSI enables designers and engineers to identify areas of weakness in their designs to hopefully factor these out. Furthermore, using FSI can provide information allowing the capture of intricacies to help pre-determine and pre-scribe the values they wish in the final design.

\section{References}

Anderson JD. 1995. Computational Fluid Dynamics - The basics with applications. (J. J. Corrigan, \& E. Castellano, Eds.) New York: McGraw-Hill.

Arioli G., \& Gazzola, F. 2017. Torsional instability in suspension bridges: The Tacoma Narrows Bridge case. Communications in Nonlinear Science and Numerical Simulation, 342357.

Bisplinghoff, R. L., Ashley, H., \& Halfman, R. L. 1955. Aeroelasticity. Addison- Wesley Pub. Co.

Crouch, I. G. 2016. The Science of Armour Materials. Kindlington: Elsevier.

Ducoin, A., \& Young, Y. L. 2013. Hydroelastic response and stability of a hydrofoil in viscous flow. Journal of Fluids and Structures, 40-57. doi:https://doi.org/10.1016/j.jfluidstructs.2012.12.0 11

Kamakoti, R., \& Shyy, W. 2004. Fluid-Structure Interaction for Aeroelastic Applications. Progress in Aerospace Sciences, 40(8),535-558. doi:https://doi.org/10.1016/j.paerosci.2005.01.001

Knight, J.J., Lucey, A.D., and Shaw, C.T., 2010. 'Fluid-structure interaction of a two-dimensional membrane in a flow with a pressure gradient with application to convertible car roofs.' J. Wind Engng $\begin{array}{llll}\text { Ind. } & \text { Aerodynamics } 98 \text { (2): } & 65-72 .\end{array}$ doi:10.1016/j.jweia.2009.09.003

Panda, C., \& SRP, V. 2009. Aeroelasticity In General and Flutter Phenomenon. Emerging Trends in Engineering and Technology (ICETET) (pp. 8185). Nagpur: IEEE. doi:10.1109/ICETET.2009.23

Ramsey, J. K. 2006. NASA Aeroelasticity Handbook. Cleveland: NASA.

Sigrist, J.-F. 2015. Fluid- Structure Interaction. Chichester: John Wilet \& Sons Ltd.

Tu, J., Yeoh, G., \& Liu, C. 2013. Computational Fluid Dynamics. Oxford: Elsevier Ltd.

Versteeg, H. K., \& Malalasekera, W. 2007. An Introduction to Computational Fluid Dynamics The Finite Volume Method (2nd ed.). Harlow: Pearson Education LTD.

Zikanov, O. 2011. Essential Computational Fluid Dynamics. John Wiley \& Sons, Incorporated. 